\title{
Elucidation of optimal proteinuria management based on the risk of ramucirumab-induced proteinuria
}

\author{
MICHIO KIMURA $^{1}$, EISEKI USAMI ${ }^{1}$, HITOMI TERAMACHI $^{2}$ and TOMOAKI YOSHIMURA ${ }^{1}$ \\ ${ }^{1}$ Department of Pharmacy, Ogaki Municipal Hospital, Ogaki, Gifu 503-8502; \\ ${ }^{2}$ Laboratory of Clinical Pharmacy, Gifu Pharmaceutical University, Gifu-shi, Gifu 501-1196, Japan
}

Received October 15, 2021; Accepted November 24, 2021

DOI: $10.3892 / \mathrm{ol} .2021 .13163$

\begin{abstract}
Proteinuria is a dose-limiting adverse effect of ramucirumab treatment, which is an anti-angiogenic agent that targets the human vascular endothelial growth factor. The predictors of proteinuria have not been completely elucidated and there is currently no consensus. The present study aimed to identify the risk factors for ramucirumab-induced proteinuria and to determine an optimal proteinuria management. A total of 145 patients who received ramucirumab at Ogaki Municipal Hospital (Ogaki, Japan) between September 2015 and March 2021 were retrospectively studied. Multivariate logistic regression analysis was conducted to evaluate the association between the patient baseline characteristics and the development of proteinuria following ramucirumab treatment. Furthermore, the time of proteinuria onset and of the worst qualitative proteinuria were recorded. Proteinuria $(>2+)$ following ramucirumab was independently associated with lung cancer [odd ratio (OR): 0.232 , 95\% confidence interval (CI): 0.061-0.874; $\mathrm{P}=0.031$ ] and proteinuria at the start of treatment [qualitative test (+/-); OR: 4.760, 95\% CI: 1.360-16.700; $\mathrm{P}=0.041]$. The median time of onset of proteinuria was 56 days (time range, 7-414 days), and the median time when the worst qualitative results were observed was 83 days (time range, 7-442 days). The $>2+$ proteinuria in the qualitative test was observed in 27 out of the 82 patients with gastric cancer $(\mathrm{P}=0.041), 8 / 21$ patients with colon cancer $(\mathrm{P}=0.188)$, and in 3 out of the 37 patients with lung cancer $(\mathrm{P}=0.003)$. The prevalence of proteinuria was low in patients with lung cancer, and proteinuria $(>2+)$ was likely to occur when the proteinuria at the start of ramucirumab was (+/-) by qualitative test. The results from the present study indicated that particular attention should be paid to proteinuria at the start of treatment when monitoring proteinuria as an adverse event of ramucirumab treatment.
\end{abstract}

Correspondence to: Mr. Michio Kimura, Department of Pharmacy, Ogaki Municipal Hospital, 4-86 Minaminokawa-cho, Ogaki, Gifu 503-8502, Japan

E-mail: kimkim0305nao@yahoo.co.jp

Key words: ramucirumab, proteinuria, risk factor, lung cancer, qualitative test

\section{Introduction}

Ramucirumab, which is an anti-angiogenic agent that targets the human vascular endothelial growth factor (VEGF) pathway, significantly improves the overall and progression-free survival in patients with colorectal, lung and gastric cancers (1-3). The use of ramucirumab is generally associated with hypertension, proteinuria and gastrointestinal bleeding (1-3). The development of angiogenesis inhibitor-induced proteinuria limits the dose of ramucirumab, and proteinuria of level $>2+$ may lead to withdrawal or discontinuation of the drug, which can therefore reduce the efficacy of the treatment (4). If the qualitative test is $\geq 2+$, a quantitative test will be performed. Proteinuria $(>2+)$ indicates that the protein is $\geq 100 \mathrm{mg} / \mathrm{dl}$.

Recently, various risk factors for proteinuria have been reported, including bevacizumab administration history (5), bevacizumab dose ( $>570 \mathrm{mg}$ ) (6), high systolic blood pressure (SBP; $\geq 130 \mathrm{mmHg}$ ) (7) and history of proteinuria (8). However, the predictors of proteinuria have not been completely elucidated and there is no consensus in this regard. Previous studies have reported the use of anti-VEGF antibody drugs, including bevacizumab or ramucirumab, in advanced colorectal cancer, and few studies have examined the differences in the development of proteinuria based on the type of cancer (5-8).

Identifying the predictors of proteinuria may help guiding future strategies for improving drug safety. Compared with placebo, East Asian (EA) and non-EA patients treated with ramucirumab had increased grade 3 or higher adverse effects (AEs), including hypertension and proteinuria (9). If grade 3 or higher AEs were observed, treatment was withdrawn. The incidence of grade 3 or higher proteinuria was 3.9 vs. $0.6 \%$ in EA vs. non-EA patients, respectively. It is therefore crucial to examine the risk factors for proteinuria in Japanese patients treated with ramucirumab.

The present study aimed to identify the risk factors for ramucirumab-induced proteinuria, and to determine an optimal proteinuria management. This study focused on the results of a qualitative test for proteinuria, as this test is simple and can be used as a reference before initiating ramucirumab treatment.

\section{Materials and methods}

Patients. We retrospectively included 189 patients who received ramucirumab at Ogaki Municipal Hospital (Ogaki, 
Japan) between September 2015 and March 2021 for gastric, colorectal, lung, liver, and esophagogastric junction cancer. We excluded 44 patients according to the following criteria: Patients who completed only one course of treatment; patients of whom urinary protein was not measured; or patients for whom urinary protein was detected at the start of ramucirumab administration. Therefore, 145 patients were included in the present study (Fig. 1). The patient characteristics were extracted from anonymized patient records. The study's retrospective protocol was approved by the institutional review board of Ogaki Municipal Hospital (Ogaki, Japan; approval no. 20210826-9), and the requirement for informed consent was waived based on the study's retrospective nature. This study was performed in accordance with the ethical standards of the 1964 Declaration of Helsinki and its later amendments.

Outcome. The primary outcome was defined as an occurrence of $>2+$ proteinuria according to the results of qualitative tests for proteinuria. Data from the first instance of the event (the highest level of proteinuria) were adopted. The qualitative test for urine protein used a test paper (measurement error caused by $\mathrm{pH}$ change). The qualitative test (1+) indicated protein levels of $30 \mathrm{mg} / \mathrm{dl}$, the qualitative test (2+) indicated protein levels of $100 \mathrm{mg} / \mathrm{dl}$ and the qualitative test (3+) indicated protein levels of $300 \mathrm{mg}$.

Management of proteinuria. Ramucirumab was administered if the qualitative test result was $<1+$. In the case of $2+$ proteinuria, the decision on ramucirumab administration was determined based on the patient's symptoms such as edema or weight gain, and condition such as presence or absence of hypoalbuminemia. A quantitative test was also performed. A 24-h whole urine test was desirable in the quantitative test; however, the urine protein/creatinine ratio was determined if the above was not possible. The urine protein/creatinine ratio is nearly equal to the daily urinary protein excretion ( $\mathrm{g} / \mathrm{day}$ ). If the urine protein was $<2 \mathrm{~g} / \mathrm{day}$, ramucirumab was continued. If the urine protein was between 2 and $3 \mathrm{~g}$, ramucirumab was withdrawn. Administration of ramucirumab was discontinued if the urine protein was $>3 \mathrm{~g} /$ day or if nephrotic syndrome occurred.

Risk factors for proteinuria. Using electronic medical records, demographic data [sex, age, height, body weight and body mass index (BMI)], indications for cancer (type of cancer and presence of any metastasis), presence of comorbidities (hypertension and diabetes mellitus), laboratory data (aspartate aminotransferase, alanine aminotransferase, total bilirubin, serum creatinine, serum sodium, serum chlorine, serum potassium), chemotherapy regimen (fluorouracil-based and platinum-based), vital signs [SBP and diastolic blood pressure (DBP)], bevacizumab or cisplatin administration history, chemotherapy treatment period before ramucirumab administration, ramucirumab administration period, proteinuria at the start of ramucirumab (+/-) and administration course were collected. The presence of hypertension was assessed based on whether antihypertensive drugs were prescribed. Clinical information was collected from the initial administration of ramucirumab until the end of the treatment.

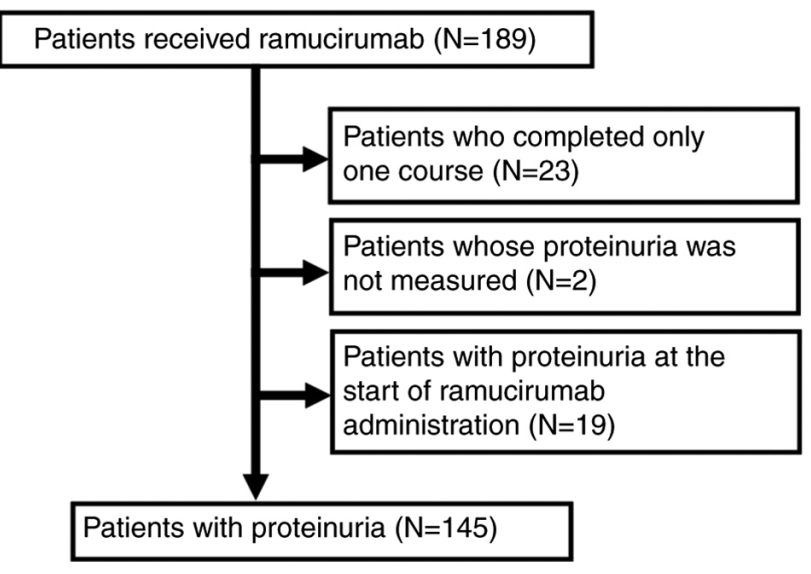

Figure 1. Flow chart of patient selection.

Proteinuria occurrence during ramucirumab treatment. The incidence of proteinuria after starting ramucirumab treatment was recorded for each type of cancer. Furthermore, the onset time and time point with the highest level of proteinuria according to the results of qualitative tests after ramucirumab administration were recorded.

Relationship between proteinuria onset date and qualitative test results $(1+-3+)$. The date of onset of proteinuria and the highest level or the first level at onset of proteinuria [qualitative test results (1+-3+)] during ramucirumab administration were recorded.

Statistical analysis. Differences between patient groups were evaluated using the Mann-Whitney U-test or Fisher's exact probability test, as appropriate. Univariate analyses were performed to evaluate the relationship between patients' baseline characteristics and the development of proteinuria. Significant variables and previously reported risk factors were entered into a multivariate logistic regression model. Optimal cut-off values for the significant variables were determined based on receiver operating characteristic (ROC) curve analyses. Spearman's correlation coefficient by rank test was used to analyze the correlation between the proteinuria onset date and the highest level of proteinuria [qualitative test results, $(1+)-(3+)] . \mathrm{P}<0.05$ was considered to indicate a statistically significant difference. All analyses were performed using EZR software (version 1.30; Saitama Medical Center, Jichi Medical University, Saitama, Japan), which is a graphical user interface for R software (The R Foundation for Statistical Computing, Vienna, Austria) (10).

\section{Results}

Patient characteristics. Patients were grouped according to their proteinuria results (2+ or higher) during ramucirumab administration. The patient's characteristics are summarized in Table I. Ramucirumab treatment lines (order of treatment) by cancer type were as follows: Gastric cancer, 2nd line (range, lines 1-4); colon cancer, 3rd line (range, lines 2-4); lung cancer, 3rd line (range, lines 1-5); and others, 2nd line (range, lines 2-3). The duration of ramucirumab administration by 

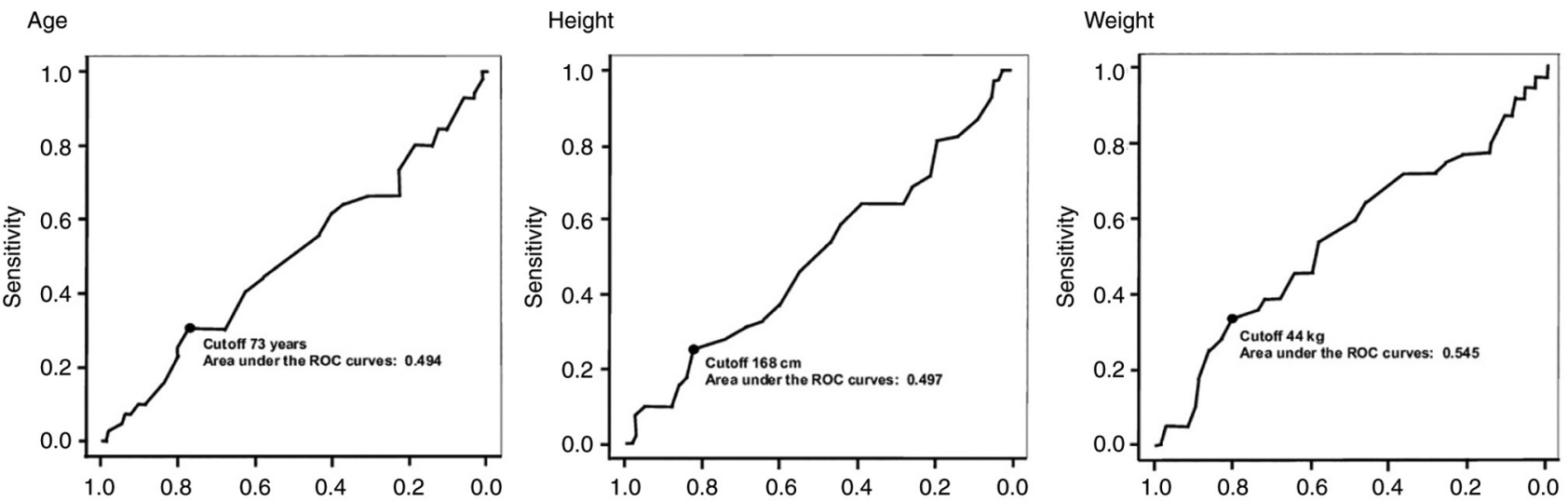

Body mass index
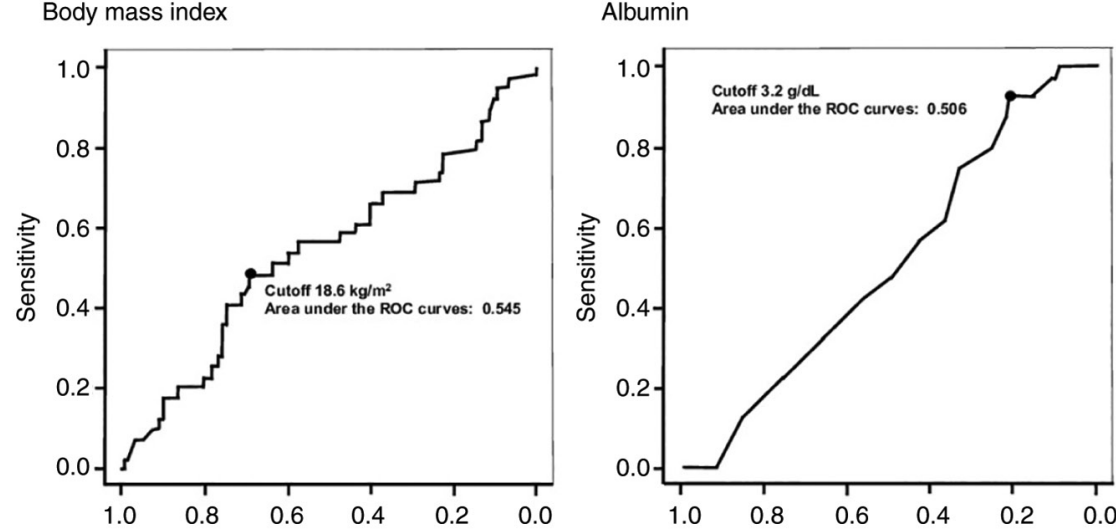

\section{Aspartate aminotransferase}

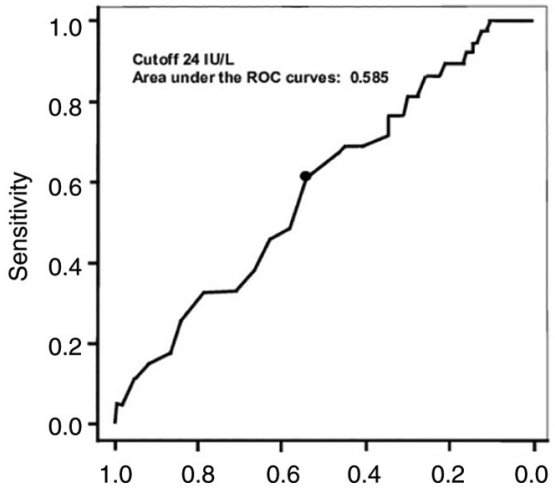

Figure 2. Area under ROC curves for age, height, weight, body mass index, albumin level and aspartate aminotransferase level. ROC, receiver operating characteristic.
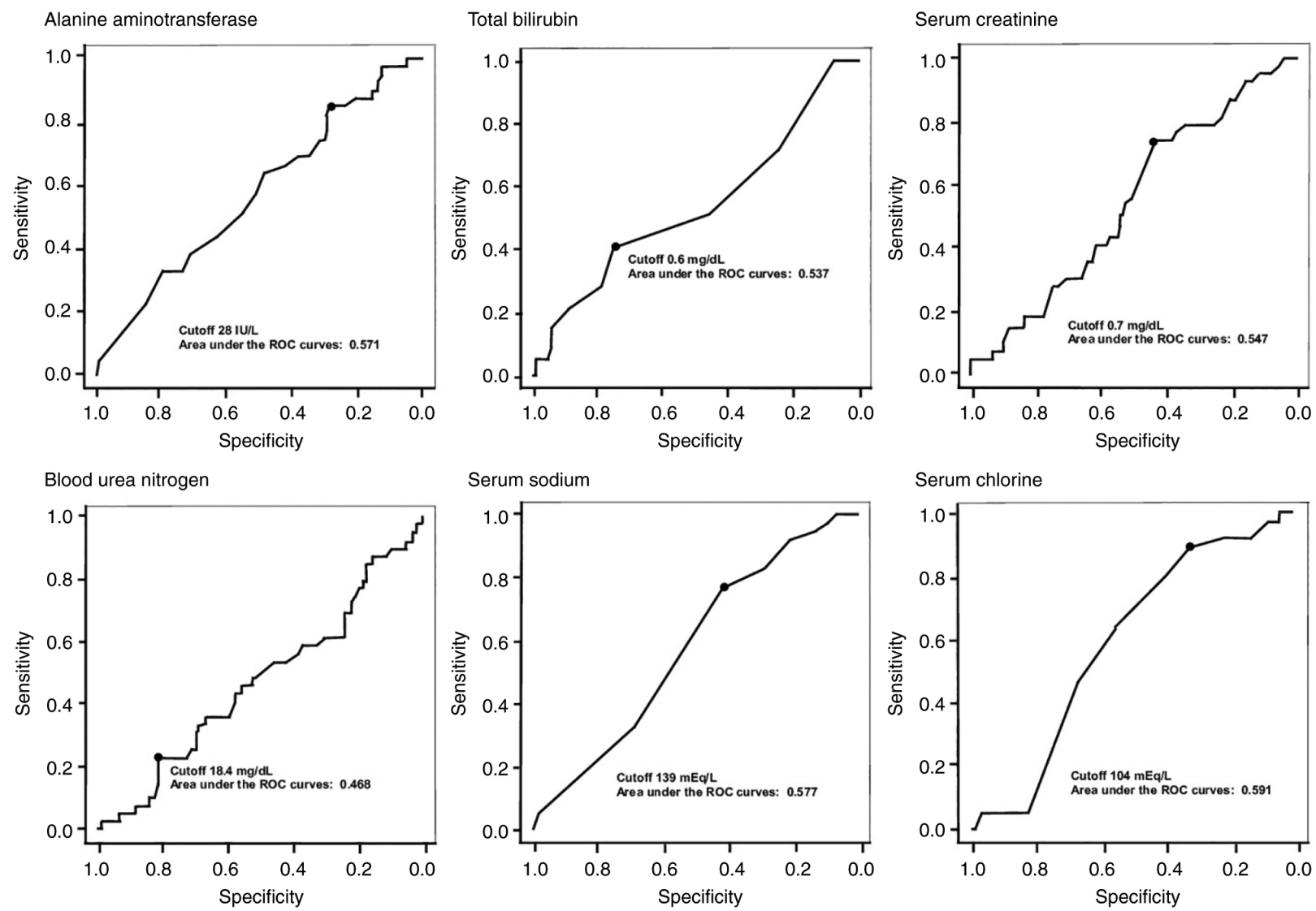

Figure 3. Area under ROC curves for alanine aminotransferase level, total bilirubin level, serum creatinine level, blood urea nitrogen level, serum sodium level and serum chlorine level. ROC, receiver operating characteristic. 
Table I. Clinicopathological characteristics of the patients.

Characteristics No proteinuria $(\mathrm{n}=107)$

Proteinuria $(\geq 2+)(n=38)$

P-value

Demographic data

Age, years, median (range)

$$
\begin{gathered}
69(35-83) \\
76 / 31 \\
162(142-185) \\
52(27-86) \\
19.9(11.7-29.1)
\end{gathered}
$$

Sex, male/female, $\mathrm{n}$

Height, cm, median (range)

Weight, kg, median (range)

Body mass index, $\mathrm{kg} / \mathrm{m}^{2}$, median (range)

Comorbidity, $\mathrm{n}$

Hypertension

Diabetes mellitus

Disease status, $\mathrm{n}$

Recurrent/Unresectable

Cancer type, $\mathrm{n}$

Lung

Gastric

Colorectal

Others

Regimen, $\mathrm{n}$

Paclitaxel plus ramucirumab

Docetaxel plus ramucirumab

FOLFIRI $^{\mathrm{b}}$ plus ramucirumab

Ramucirumab

Vital signs before administration

SBP, mmHg, median (range)

DBP, mmHg, median (range)

Laboratory test values before chemotherapy

Aspartate aminotransferase, IU/1

Alanine aminotransferase, IU/1

Serum creatinine, $\mathrm{mg} / \mathrm{dl}$

Total bilirubin, $\mathrm{mg} / \mathrm{dl}$

Albumin, g/dl

Serum sodium, $\mathrm{mEq} / \mathrm{l}$

Serum chlorine, $\mathrm{mEq} / \mathrm{l}$

Serum potassium, $\mathrm{mEq} / \mathrm{l}$

Blood urea nitrogen, $\mathrm{mg} / \mathrm{dl}$

Metastatic site, $\mathrm{n}$

Liver

Lung

Lymph node

Peritoneal

Bone

Brain

Others

Administration history, $\mathrm{n}$

Bevacizumab

Cisplatin

Treatment period

Chemotherapy treatment period before ramucirumab

administration, days median (range)

Ramucirumab administration period, days, median (range)

$$
30
$$

11

$34 / 73$

34

55

14

4

56

34

14

3

$120(81-160)$

$25(11-132)$

17 (6-130)

$0.73(0.36-1.6)$

$0.5(0.2-1.6)$

$3.7(1.8-5.0)$

139 (125-144)

$105(92-114)$

$4.2(3.0-6.0)$

$13.6(5.4-30.6)$

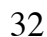

9

32

4

7

6

10

12

61

$246(0-1891)$

119 (14-1479)
70 (45-106)

$\begin{array}{cc}69(39-81) & 0.755 \\ 23 / 15 & 0.232 \\ 162(146-179) & 0.984 \\ 50(35-101) & 0.453 \\ 19.2(14.5-31.5) & 0.462\end{array}$

0.133

0.364

0.249

$0.004^{\mathrm{a}}$

$0.036^{\mathrm{a}}$

0.239

0.227

$<0.001^{\mathrm{a}}$

$<0.001^{\mathrm{a}}$

0.239

0.297

126 (91-168)

0.098

74 (53-97)

0.416

$23.5(11-61)$

0.118

$15.5(6-71)$

0.195

$0.76(0.5-1.89)$

0.510

$0.5(0.3-1.5)$

0.417

$3.7(2.7-4.2)$

0.973

140 (134-144)

0.268

$106(98-111)$

0.136

$4.1(3.3-5.4)$

0.666

$13.6(4.0-29.3)$

0.545

$0.011^{\mathrm{a}}$

0.893

0.383

$<0.001^{\mathrm{a}}$

0.499

0.293

0.646

0.130

0.509

24

0.707

$272.5(0-1673)$

$0.004^{\mathrm{a}}$ 
Table I. Continued.

\begin{tabular}{|c|c|c|c|}
\hline Characteristics & No proteinuria $(\mathrm{n}=107)$ & Proteinuria $(\geq 2+)(n=38)$ & P-value \\
\hline \multicolumn{4}{|l|}{ Others } \\
\hline Proteinuria at the start of ramucirumab $(+/-)$, yes/no, $n$ & 9/98 & $9 / 29$ & $0.001^{\mathrm{a}}$ \\
\hline Number of cycles of ramucirumab, median (range) & $4(2-20)$ & $7(2-29)$ & $0.014^{\mathrm{a}}$ \\
\hline
\end{tabular}

SBP, systolic blood pressure; DBP, diastolic blood pressure. ${ }^{\mathrm{a}} \mathrm{P}<0.05$. ${ }^{\mathrm{b}} \mathrm{FOLFIRI}$, combination of fluorouracil/leucovorin and irinotecan.

Table II. Proteinuria according to cancer type in patients treated with ramucirumab.

\begin{tabular}{lcccc}
\hline & & \multicolumn{2}{c}{ Number of patients with proteinuria, $\mathrm{n}(\%)$} & \\
\cline { 3 - 4 } Type of cancer & Number & Proteinuria & Proteinuria $(\geq 2+)$ & P-value $^{\mathrm{a}}$ \\
\hline Gastric cancer & 82 & $37(45.1)$ & $27(32.9)$ & $0.041^{\mathrm{b}}$ \\
Colorectal cancer & 22 & $12(54.5)$ & $8(36.3)$ & 0.188 \\
Lung cancer & 37 & $7(18.9)$ & $3(8.1)$ & $0.003^{\mathrm{b}}$ \\
Others & 4 & $1(25.0)$ & 0 & 0.292 \\
\hline
\end{tabular}

${ }^{\text {aP }}$-value: Proteinuria $(\geq 2+)$ vs. proteinuria $(\leq 1+)$. ${ }^{\text {}} \mathrm{P}<0.05$.

cancer type was as follows: Gastric cancer, 143 days (range, 14-910 days); colon cancer, 158 days (range, 21-1479 days); lung cancer, 114 days (range, 15-472 days); and others, 144 days (range, 27-166 days). Patients with lung cancer (114 days) had a significantly shorter ramucirumab administration period than patients with other types of cancer (154 days) $(\mathrm{P}=0.043)$.

Development of proteinuria according to ramucirumab treatment. Patients were grouped according to whether they did or did not develop proteinuria (38 and 107 patients, respectively). Proteinuria was observed in 37 out of the 82 patients with gastric cancer, 12 out of the 22 patients with colon cancer, 7 out of the 37 patients with lung cancer and in 1 out of the 4 patients with other types of cancer. A score of $\geq 2+$ for proteinuria was observed in 27 out of the 82 patients with gastric cancer $(\mathrm{P}=0.041)$, in 8 out of the 22 colon patients with cancers $(\mathrm{P}=0.188)$, in 3 out of the 37 patients with lung cancer $(\mathrm{P}=0.003)$ and in 0 out of the 4 patients with other types of cancer $(\mathrm{P}=0.292$; Table II $)$. The onset of proteinuria was observed in a median of 56 days (range, 7-414 days) and the worst qualitative results were observed in 83 days (range, 7-442 days).

Risk factors for proteinuria after ramucirumab treatment. Tables III and IV show the results of the univariate and multivariate logistic regression analyses regarding the risk factors for the development of proteinuria following ramucirumab treatment. Proteinuria was independently associated with lung cancer [odds ratio (OR): $0.232,95 \%$ confidence interval (CI): $0.061-0.874 ; \mathrm{P}=0.031]$ and proteinuria at the start of treatment (+/-; OR: 4.760, 95\% CI: 1.360-16.700; $\mathrm{P}=0.041$ ).

According to the results from ROC curve analysis, the cut-off value and the area under the ROC curves (95\% CI) of certain factors were as follows: Age, 73 years, 0.494 (0.383-0.605); height, $168 \mathrm{~cm} 0.497$ (0.387-0.608); weight, $44 \mathrm{~kg}, 0.545$ (0.433-0.657); BMI, $18.6 \mathrm{~kg} / \mathrm{m}^{2}, 0.545$ (0.434-0.656); albumin, $3.2 \mathrm{~g} / \mathrm{dl}, 0.506$ (0.406-0.606); aspartate aminotransferase, 24 IU/1, 0.585 (0.482-0.688); alanine aminotransferase, $28 \mathrm{IU} / 1,0.571$ (0.465-0.676); total bilirubin, $0.6 \mathrm{mg} / \mathrm{dl}, 0.537$ (0.426-0.647); serum creatinine, $0.7 \mathrm{mg} / \mathrm{dl}, 0.547$ (0.445-0.649); blood urea nitrogen, $18.4 \mathrm{mg} / \mathrm{dl}, 0.468$ (0.360-0.576); serum sodium, $139 \mathrm{mEq} / 1,0.577$ (0.478-0.676); serum chlorine, $104 \mathrm{mEq} / 1,0.591$ (0.495-0.686); serum potassium, $4.1 \mathrm{mEq} / \mathrm{l}$, 0.534 (0.428-0.640); SBP, $128 \mathrm{mmHg}, 0.607$ (0.499-0.715); chemotherapy treatment period before ramucirumab administration, 223 days, 0.504 (0.395-0.612; Figs. 2-4).

Relationship between the time of onset of proteinuria and the qualitative test results $(1+-3+)$. The onset of proteinuria occurred early after ramucirumab administration. The likelihood of proteinuria did not increase with the increased duration of the treatment. Fig. 5A shows that the proteinuria onset date and the highest level of proteinuria (qualitative test results, 1+-3+) were not associated, based on the highest value observed during the ramucirumab administration period (correlation coefficient; rs=0.175, P=0.144). Similarly, Fig. 5B shows that the proteinuria onset date and the proteinuria level at onset (qualitative test results, 1+-3+) were not associated, based on the first value obtained during the ramucirumab administration period ( $\mathrm{rs}=0.177, \mathrm{P}=0.227$ ).

\section{Discussion}

The present study aimed to identify risk factors for proteinuria induced by ramucirumab, and investigated whether there could be differences in the likelihood of proteinuria according 
Table III. Univariate logistic regression analysis of the risk factors for ramucirumab-related proteinuria.

\begin{tabular}{|c|c|c|c|}
\hline Factors & Odds ratio & $95 \%$ confidence interval & P-value \\
\hline \multicolumn{4}{|l|}{ Age, years } \\
\hline$>73$ & 1.520 & $0.670-3.440$ & 0.317 \\
\hline \multicolumn{4}{|l|}{ Sex } \\
\hline Male & 0.661 & $0.307-1.430$ & 0.292 \\
\hline \multicolumn{4}{|l|}{ Height, cm } \\
\hline$>168$ & 1.580 & $0.659-3.780$ & 0.305 \\
\hline \multicolumn{4}{|l|}{ Weight, kg } \\
\hline$>44$ & 0.523 & $0.227-1.210$ & 0.129 \\
\hline \multicolumn{4}{|c|}{ Body mass index, $\mathrm{kg} / \mathrm{m}^{2}$} \\
\hline$>18.6$ & 0.527 & $0.249-1.120$ & 0.095 \\
\hline \multicolumn{4}{|l|}{ Albumin, $\mathrm{g} / \mathrm{dl}$} \\
\hline$>3.2$ & 3.140 & $0.884-11.200$ & 0.077 \\
\hline \multicolumn{4}{|c|}{ Aspartate aminotransferase, IU/1 } \\
\hline$>24$ & 0.777 & $0.372-1.620$ & 0.501 \\
\hline \multicolumn{4}{|c|}{ Alanine aminotransferase, IU/1 } \\
\hline$>28$ & 0.529 & $0.211-1.330$ & 0.175 \\
\hline \multicolumn{4}{|c|}{ Total bilirubin, $\mathrm{mg} / \mathrm{dl}$} \\
\hline$>0.6$ & 2.040 & $0.939-4.410$ & 0.072 \\
\hline \multicolumn{4}{|c|}{ Serum creatinine, $\mathrm{mg} / \mathrm{dl}$} \\
\hline$>0.7$ & 1.990 & $0.831-4.780$ & 0.122 \\
\hline \multicolumn{4}{|c|}{ Blood urea nitrogen, $\mathrm{mg} / \mathrm{dl}$} \\
\hline$>18.4$ & 1.290 & $0.530-3.140$ & 0.575 \\
\hline \multicolumn{4}{|c|}{ Serum sodium, mEq/l } \\
\hline$>139.0$ & 1.420 & $0.657-3.060$ & 0.373 \\
\hline \multicolumn{4}{|c|}{ Serum chlorine, $\mathrm{mEq} / \mathrm{l}$} \\
\hline$>104$ & 2.160 & $0.866-5.380$ & 0.098 \\
\hline \multicolumn{4}{|c|}{ Serum potassium, mEq/l } \\
\hline$>4.1$ & 0.753 & $0.357-1.590$ & 0.457 \\
\hline \multicolumn{4}{|c|}{ Systolic blood pressure, $\mathrm{mmHg}$} \\
\hline$>128$ & 0.661 & $0.307-1.430$ & 0.292 \\
\hline \multicolumn{4}{|c|}{ Bevacizumab administration history } \\
\hline Yes & 2.020 & $0.757-5.400$ & 0.160 \\
\hline \multicolumn{4}{|c|}{ Cisplatin administration history } \\
\hline Yes & 1.180 & $0.557-2.500$ & 0.665 \\
\hline \multicolumn{4}{|c|}{$\begin{array}{l}\text { Chemotherapy treatment period before ramucirumab } \\
\text { administration, days }\end{array}$} \\
\hline$>223$ & 1.320 & $0.626-2.800$ & 0.463 \\
\hline \multicolumn{4}{|l|}{ With diabetes } \\
\hline Yes & 1.570 & $0.538-4.580$ & 0.409 \\
\hline \multicolumn{4}{|l|}{ Cancer type } \\
\hline Lung cancer & 0.176 & $0.051-0.614$ & $0.006^{\mathrm{a}}$ \\
\hline \multicolumn{4}{|c|}{ Proteinuria at the start of ramucirumab treatment $(+/-)$} \\
\hline Yes & 4.220 & $1.530-11.700$ & $0.006^{\mathrm{a}}$ \\
\hline \multicolumn{4}{|c|}{ Taking antihypertensive drugs } \\
\hline Yes & 0.581 & $0.231-1.460$ & 0.248 \\
\hline
\end{tabular}

Results of univariate logistic regression analyses based on binary response model. ${ }^{\mathrm{a}} \mathrm{P}<0.05$. 
Table IV. Multivariate logistic regression analysis of the risk factors for ramucirumab-related proteinuria $(\geq 2+)$.

\begin{tabular}{|c|c|c|c|}
\hline Factors & Odds ratio & $95 \%$ confidence interval & P-value \\
\hline \multicolumn{4}{|c|}{ Bevacizumab administration history } \\
\hline Yes & 1.450 & $0.440-4.770$ & 0.541 \\
\hline \multicolumn{4}{|l|}{ Cancer type } \\
\hline Lung cancer & 0.232 & $0.061-0.874$ & $0.031^{\mathrm{a}}$ \\
\hline \multicolumn{4}{|c|}{ Proteinuria at the start of ramucirumab treatment $(+/-)$} \\
\hline Yes & 4.760 & $1.360-16.700$ & $0.041^{\mathrm{a}}$ \\
\hline \multicolumn{4}{|c|}{ Systolic blood pressure, $\mathrm{mmHg}$} \\
\hline$>128$ & 1.920 & $0.806-4.590$ & 0.141 \\
\hline \multicolumn{4}{|c|}{ Antihypertensive drugs } \\
\hline Yes & 0.556 & $0.180-1.710$ & 0.306 \\
\hline \multicolumn{4}{|c|}{ Body mass index, $\mathrm{kg} / \mathrm{m}^{2}$} \\
\hline$>18.6$ & 0.720 & $0.301-1.720$ & 0.460 \\
\hline \multicolumn{4}{|l|}{ Albumin, g/dl } \\
\hline$>3.2$ & 3.470 & $0.884-13.600$ & 0.075 \\
\hline \multicolumn{4}{|l|}{ Diabetes } \\
\hline Yes & 1.840 & $0.547-6.200$ & 0.324 \\
\hline
\end{tabular}

Results of multivariate logistic regression analysis based on binary response model for variables extracted by forward selection. ${ }^{\text {a }}<0.05$.
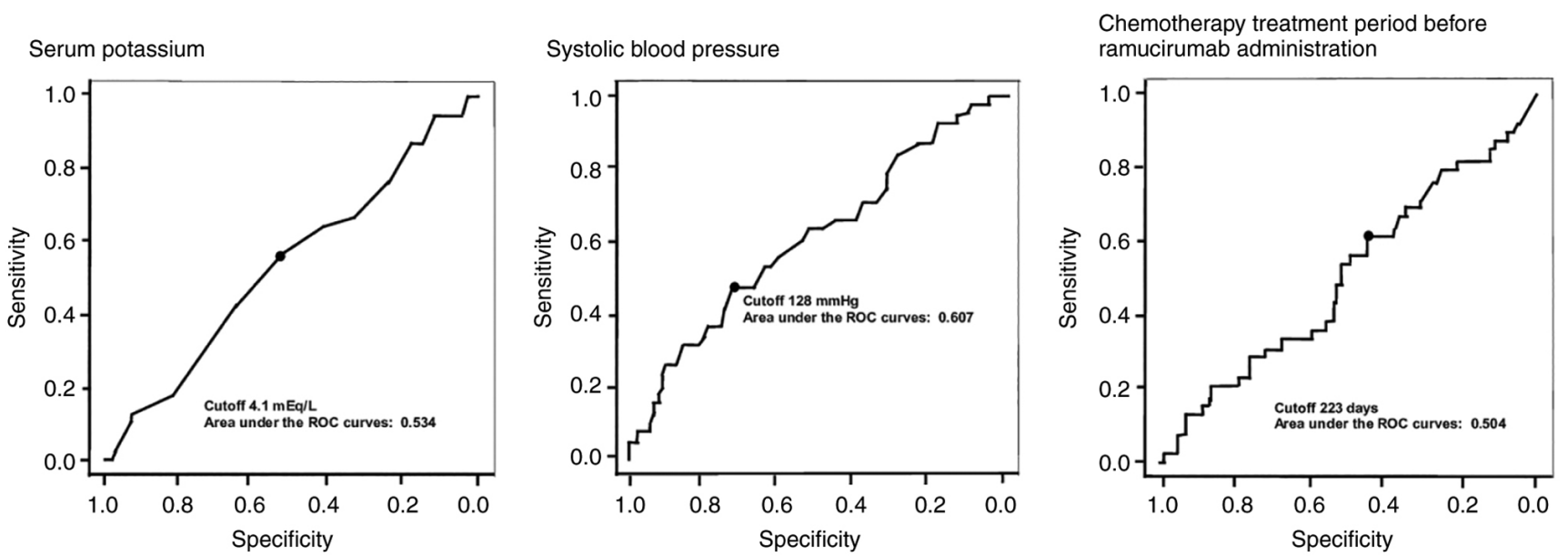

Figure 4. Area under ROC curves for serum potassium level, systolic blood pressure and chemotherapy treatment period before ramucirumab administration. ROC, receiver operating characteristic.

to the type of cancer. The results demonstrated that the type of cancer (lung cancer) and urinary protein levels (+/-) at the start of ramucirumab therapy were associated with proteinuria $(\geq 2+)$ in patients with cancer treated with ramucirumab. In clinical practice, the incidence of proteinuria is low in the case of lung cancer, and $>2+$ proteinuria is likely to occur when the proteinuria at the start of ramucirumab is +/-. In the present study, we observed that the possibility of proteinuria was not related to bevacizumab administration history, co-administration of paclitaxel with ramucirumab, or SBP at the start of ramucirumab treatment.

Previous studies reported the risk factors for ramucirumab-induced proteinuria, including bevacizumab administration history, cumulative bevacizumab dose, high
SBP, and history of proteinuria (5-8). Although a relationship between bevacizumab administration history and proteinuria was reported by Tanaka et al (5) and Kobayashi et al (8), such relationship was not demonstrated in the present study. Differences in the types of cancer studied and the number of patients treated with bevacizumab may have led to the lack of agreement with these two studies. The study by Kobayashi et al (8) was limited to patients with advanced colorectal cancer.

Based on the results from the present study, it was not possible to determine whether bevacizumab administration history had an effect on proteinuria, because proteinuria was highly prevalent in patients with gastric cancer and in patients with colorectal cancer with a history of bevacizumab 
A

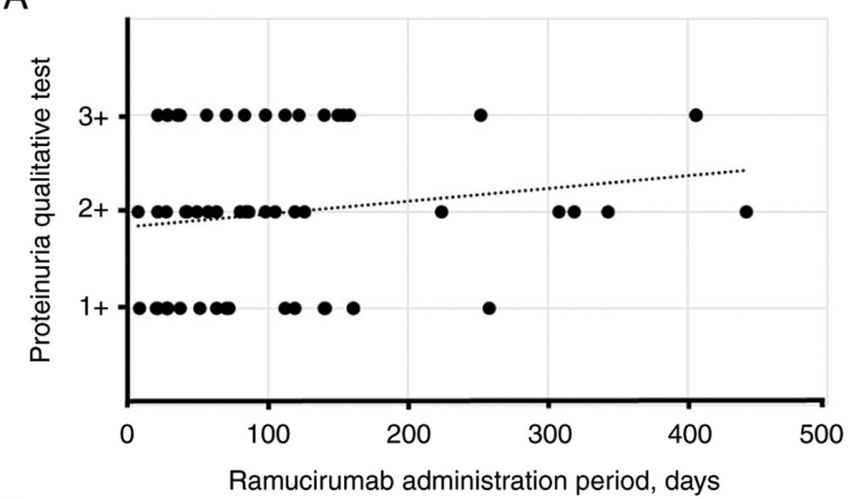

B

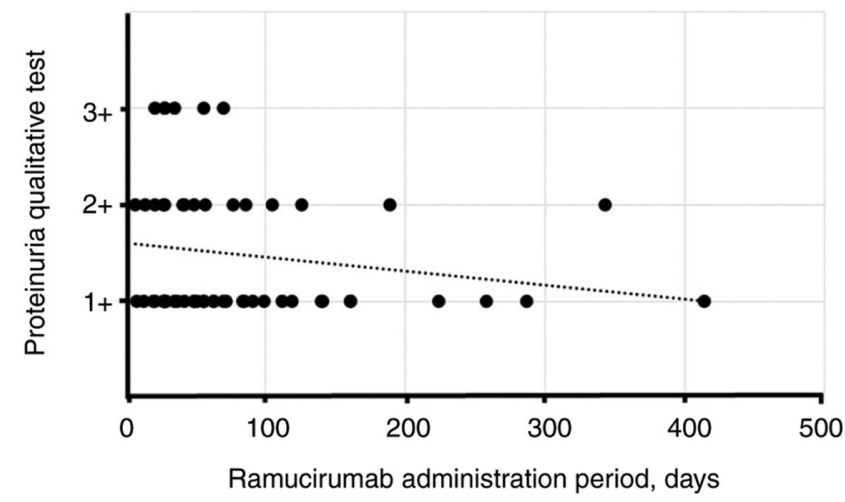

Figure 5. Relationship between the proteinuria onset date and the highest level of proteinuria [qualitative test results $(1+-3+)$ ]. (A) Proteinuria onset date and highest proteinuria level [qualitative test results (1+-3+)]. (B) Proteinuria onset date and first proteinuria level [qualitative test results $(1+-3+)]$.

administration. Cisplatin is often used in patients with gastric cancer. Therefore, it was predicted that impaired renal function would affect proteinuria, but no such effect was observed in the present study. This may be due to hydration status and renal protection during cisplatin administration. The simultaneous use of paclitaxel and ramucirumab has been reported as a risk factor for decreased renal function $(11,12)$. Therefore, proteinuria is likely to occur upon administering paclitaxel with ramucirumab. However, in the present study, the combination of paclitaxel with ramucirumab in patients with gastric cancer was not identified as a risk factor for proteinuria.

It has been reported that the total dose of bevacizumab is a risk factor for proteinuria $(6,13)$. The target drugs evaluated by Kanbayashi et al (13) were bevacizumab, ramucirumab and aflibercept, which may be a factor contributing to this observation. However, despite drug differences, the total dose was not a risk factor in the present study. It was also clear from the onset time that the proteinuria was not related to the total dose of the drug. Proteinuria is often induced early during ramucirumab treatment, as reported by Kobayashi et al (8) as well. In agreement with the above, proteinuria induction and worst grade proteinuria were observed from an early stage in the present study. However, the onset of proteinuria was also observed late after ramucirumab administration in some cases.

SBP has been reported to be a risk factor for proteinuria $(6,7,13,14)$, although it was not identified as a risk factor in the present study. There was no difference in SBP between patients with and without proteinuria. This may have been influenced by the difference in the target drugs. In the present study, we did not collect data on the types of antihypertensive agents used, which may have affected the results. However, in agreement with our study, Hirai et al (15) did not identify SBP as a risk factor for proteinuria (15).

Kobayashi et al (8) listed history of proteinuria as a risk factor of proteinuria. However, this study was conducted in patients with colorectal cancer. In the present study, proteinuria status of $+/-$ at the start of ramucirumab treatment was demonstrated to be a risk factor for $>2+$ proteinuria. Subsequently, it can be hypothesized that patients who already have proteinuria at the start of ramucirumab may be at high risk of exacerbation.

Hirai et al (15) reported that colorectal cancer is a risk factor for proteinuria in ramucirumab- and bevacizumab-treated patients. In the present study, colorectal cancer was not identified as a risk factor of proteinuria, and lung cancer was shown to reduce the risk of proteinuria. The incidence of proteinuria was also high in patients with gastric cancer. However, it has been reported that the incidence of proteinuria is high in patients with lung cancer. In the RELAY study where erlotinib plus ramucirumab was investigated (2), the incidence of proteinuria was 33.9 vs. $8.4 \%$ (erlotinib plus ramucirumab groups vs. untreated groups), which was higher than that for other types of cancer, as reported by the RAINBOW study that evaluated gastric cancer (3) (16.5 vs. 6.1\%) and compared the ramucirumab-treated vs. untreated groups. Such a high proteinuria rate may be attributed to the fact that the RELAY study evaluated the drug as 1st line therapy and that the treatment period was long. In the present study, ramucirumab was evaluated as the median 3rd line of treatment in patients with lung cancer. The median onset time was 165.5 days. However, it should be noted that in the present study, the dose used was $10 \mathrm{mg} / \mathrm{kg}$ every 2 weeks, which is higher than the dose used in clinical practice. In the current study, lung cancer was associated with a reduced risk of proteinuria. The ramucirumab dose in patients with lung cancer was $10 \mathrm{mg} / \mathrm{kg}$ every 3 weeks, whereas it was $8 \mathrm{mg} / \mathrm{kg}$ every 2 weeks in patients with gastric cancer and colon cancer. The low risk of proteinuria in patients with lung cancer may have been due to the difference in ramucirumab dose or due to the short duration of ramucirumab administration.

There are numerous reports on the relationship between obesity and proteinuria (16-18). Hirai et al (15) reported a $\mathrm{BMI} \geq 24$ as a risk factor. However, BMI was not a risk factor in the present study. Furthermore, there was no difference in BMI between patients with and without proteinuria. It is possible that $\mathrm{BMI} \geq 24$ could not be detected as a risk factor because the incidence of obesity in the current study was low [23/145 (15.8\%)]. Rosenstock et al (17) reported that diabetic patients have a significantly higher prevalence of proteinuria and albuminuria than non-diabetic patients, although in that study, the BMI of diabetic patients was not different from that of non-diabetic patients.

Proteinuria has been reported to have a poor prognosis $(19,20)$. In the present study, proteinuria induction was not related to prognosis (data not shown). The reason for this remains unclear, and additional patient characteristics may potentially serve crucial roles in the development of proteinuria. 
The following points can be considered as future strategies for improving safety. A 24-h whole urine test using livestock urine is desirable for proteinuria testing, although this test is often difficult to perform in all cases. In addition, in the case of a $2+$ result in the qualitative test, performing a quantitative test later may lead to a time lag. Proteinuria is observed from the early stage of ramucirumab treatment. Therefore, if the urinary protein at the start of treatment is +/-in a qualitative test, a quantitative test should be performed at an early stage. In addition, patients who already have proteinuria at the start of ramucirumab are more likely to discontinue treatment due to severe proteinuria, thus this should be taken into account when deciding on the anticancer drug to choose.

One limitation of the present study is that the findings are only preliminary and need to be confirmed in larger randomized controlled trials. In addition, urinary protein was assessed using a qualitative rather than a quantitative test. It is possible that the drug may not be withdrawn in clinical practice in all cases. However, because qualitative studies can predict proteinuria induction after ramucirumab initiation, the current findings could be applied to formulate anti-angiogenic treatment strategies with improved safety and efficacy. This would also help to improve the patient's quality of life.

In summary, it is crucial to respond to the onset of proteinuria from an early stage to ensure that ramucirumab therapy can be continued. Physicians and pharmacists should pay particular attention to proteinuria at the start of treatment when monitoring proteinuria as a side effect of ramucirumab treatment.

\section{Acknowledgements}

Not applicable.

\section{Funding}

No funding was received.

\section{Availability of data and materials}

The datasets used and/or analyzed during the current study are available from the corresponding author on reasonable request.

\section{Authors' contributions}

MK contributed to the study design and collected the data. EU, HT and TY contributed to the study design, reviewed the article and supervised the drafting of the manuscript and the submission process. All authors read and approved the final manuscript. MK and EU confirm the authenticity of all the raw data.

\section{Ethics approval and consent to participate}

All procedures in this study involving human participants were performed in accordance with the ethical standards of the institutional and/or national research committee and with the 1964 Declaration of Helsinki and its later amendments. The study's retrospective protocol was approved by the institutional review board of Ogaki Municipal Hospital (Ogaki,
Japan; approval no. 20210826-9). The need for formal consent was waived owing to the study design.

\section{Patient consent for publication}

Not applicable.

\section{Competing interests}

The authors declare that they have no competing interests.

\section{References}

1. Tabernero J, Yoshino T, Cohn AL, Obermannova R, Bodoky G, Garcia-Carbonero R, Ciuleanu TE, Portnoy DC, Van Cutsem E, Grothey A, et al: Ramucirumab versus placebo in combination with second-line FOLFIRI in patients with metastatic colorectal carcinoma that progressed during or after first-line therapy with bevacizumab, oxaliplatin, and a fluoropyrimidine (RAISE): A randomised, double-blind, multicentre, phase 3 study. Lancet Oncol 16: 499-508, 2015.

2. Nakagawa K, Garon EB, Seto T, Nishio M, Ponce Aix S Paz-Ares L, Chiu CH, Park K, Novello S, Nadal E, et al: Ramucirumab plus erlotinib in patients with untreated, EGFR-mutated, advanced non-small-cell lung cancer (RELAY): A randomised, double-blind, placebo-controlled, phase 3 trial. Lancet Oncol 20: 1655-1669, 2019.

3. Wilke H, Muro K, Van Cutsem E, Oh SC, Bodoky G, Shimada Y, Hironaka S, Sugimoto N,Lipatov O, Kim TY, et al: Ramucirumab plus paclitaxel versus placebo plus paclitaxel in patients with previously treated advanced gastric or gastro-oesophageal junction adenocarcinoma (RAINBOW): A double-blind, randomised phase 3 trial. Lancet Oncol 15: 1224-1235, 2014.

4. Abdel-Rahman $\mathrm{O}$ and ElHalawani $\mathrm{H}$ : Proteinuria in patients with solid tumors treated with ramucirumab: A systematic review and meta-analysis. Chemotherapy 60: 325-333, 2014.

5. Tanaka T, Kurata Y, Takase N, Himoto M, Shinmen T, Dan K, Kajizono M, Masaoka Y, Nakamoto A, Nawa H, et al: Risk factor of proteinuria in patients receiving ramucirumab. Iryo Yakugaku 47: 250-255, 2021 (In Japanese).

6. Sobue N, Iwai M, Usami E, Okada K, Kimura M, Nakao T, Yoshimura $\mathrm{T}$ and Nishijima T: Analysis of the risk factors for onset of proteinuria with bevacizumab administration and the effect of renin-angiotensin system depressant drugs. Iryo Yakugaku 42: 381-386, 2016 (In Japanese).

7. Teramachi H, Shiga H, Komada N, Tamura K, Yasuda M, Umeda M, Tachi T, Goto C and Tsuchiya T: Risk factors contributing to urinary protein expression resulting from bevacizumab combination chemotherapy. Pharmazie 68: 217-220, 2013.

8. Kobayashi T, Tanizawa Y, Nagaoka S, Chen Y, Kitagawa N, Muto $M$ and Piao Y: Incidence of proteinuria among Japanese colorectal cancer patients after receiving ramucirumab-cohort study using claim database. Gan To Kagaku Ryoho 47: 917-922, 2020 (In Japanese).

9. Yen CJ, Muro K, Kim TW, Kudo M, Shih JY, Lee KW, Chao Y, Kim SW, Yamazaki K, Sohn J, et al: Ramucirumab safety in East Asian patients: A meta-analysis of six global, randomized, double-blind, placebo-controlled, phase III clinical trials. J Glob Oncol 4: 1-12,2018.

10. Kanda Y: Investigation of the freely available easy-to-use software 'EZR' for medical statistics. Bone Marrow Transplant 48: 452-458, 2013.

11. Thigpen JT, Blessing JA, Ball H, Hummel SJ and Barrett RJ: Phase II trial of paclitaxel in patients with progressive ovarian carcinoma after platinum-based chemotherapy: A gynecologic oncology group study. J Clin Oncol 12: 1748-1753, 1994.

12. de Jongh FE, van Veen RN, Veltman SJ, de Wit R, van der Burg ME, van den Bent MJ, Planting AS, Graveland WJ, Stoter $\mathrm{G}$ and Verweij J: Weekly high-dose cisplatin is a feasible treatment option: Analysis on prognostic factors for toxicity in 400 patients. Br J Cancer 88: 1199-1206, 2003.

13. Kanbayashi Y, Ishikawa T, Tabuchi Y, Sakaguchi K, Ouchi Y, Otsuji E, Takayama K and Taguchi T: Predictive factors for the development of proteinuria in cancer patients treated with bevacizumab, ramucirumab, and aflibercept: A single-institution retrospective analysis. Sci Rep 10: 2011, 2020. 
14. Shibata M, Sato KK, Uehara S, Koh H, Kinuhata S, Oue K Kambe H, Morimoto M and Hayashi T: Blood pressure components and the risk for proteinuria in Japanese men: The Kansai healthcare study. J Epidemiol 27: 505-510, 2017.

15. Hirai T, Shuji Y, Takiyama M, Hanada K and Itoh T: Renin-angiotensin system inhibitors for countering proteinuria induced by angiogenesis inhibitors: A retrospective observational analysis. Cancer Chemother Pharmacol 84: 195-202, 2019.

16. Lafayette RA, McCall B, Li N, Chu L, Werner P, Das A and Glassock R: Incidence and relevance of proteinuria in bevacizumab-treated patients: Pooled analysis from randomized controlled trials. Am J Nephrol 40: 75-83, 2014.
17. Rosenstock JL, Pommier M, Stofels G, Patel S and Michelis MF: Prevalence of proteinuria and albuminuria in an obese population and associated risk factors. Front Med (Lausanne) 5: 122, 2018

18. Kim JK, Ju YS, Moon SJ, Song YR, Kim HJ and Kim SG: High pulse pressure and metabolic syndrome are associated with proteinuria in young adult women. BMC Nephrol 14: 45, 2013.

19. Yang Y, Li HY, Zhou Q, Peng ZW, An X, Li W, Xiong LP, Yu XQ, Jiang WQ and Mao HP: Renal function and all-cause mortality risk among cancer patients. Medicine (Baltimore) 95: e3728, 2016.

20. Kim MJ, Kang YU, Kim CS, Choi JS, Bae EH, Ma SK, Kweon SS and Kim SW: Proteinuria as a risk factor for mortality in patients with colorectal cancer. Yonsei Med J 54: 1194-1201, 2013. 